Thayer, T., Tejada, J. \& Murillo, A. (2021). La formación tecnológica del profesorado de música en educación secundaria. Un estudio de intervención basado en la integración de contenidos musicales, tecnológicos y pedagógicos. Revista Electrónica Interuniversitaria de Formación del Profesorado, 24(3), 1-20.

\title{
La formación tecnológica del profesorado de música en educación secundaria. Un estudio de intervención basado en la integración de contenidos musicales, tecnológicos y pedagógicos
}

Tomás Thayer ${ }^{1}$, Jesús Tejada ${ }^{2}$, Adolf Murillo ${ }^{2}$

(1) Universidad Austral de Chile (2) Universidad de Valencia

\section{Resumen}

El presente trabajo de carácter descriptivo-exploratorio forma parte de un trabajo de mayor alcance realizado en Chile y España. Su objetivo ha sido fortalecer la formación tecnológica de los futuros profesores de música en educación secundaria mediante el diseño, la implementación y la evaluación de un módulo de materia en dos cohortes consecutivas (cohortes 2017 y 2018, n= 81) de un máster profesional. En el diseño de intervención se tuvo en cuenta varios componentes de alfabetización tecnológica y dos estrategias de aprendizaje: proyectos (ABP) y trabajo cooperativo. La novedad del diseño ha consistido en la adaptación de un marco teórico de integración de contenidos disciplinares-tecnológicospedagógicos denominado TPACK que intenta favorecer la comprensión de la tecnología y su aplicación en la práctica docente de aula. Los productos de formación, objetos de los proyectos de trabajo, fueron materiales didácticos aplicables en el aula de educación secundaria. Los resultados de ambas cohortes sugieren que los componentes caracterizadores de la intervención pueden ayudar a planificar de forma más reflexiva el uso de la tecnología musical en el aula. Además, el alumnado afirma que mejoró la autopercepción sobre sus habilidades tecnológicas en situaciones docentes y que dotaron de significado a la tecnología en su profesión.

\section{Palabras clave}

formación del profesorado; educación musical; tecnología musical; integración de contenidos pedagógicos y tecnológicos; aprendizaje basado en proyectos.

\section{Contacto:}

Tomás Thayer-Morel, tomas.thayer@uach.cl, Facultad de Arquitecturas y Arte. Araucarias 455, Valdivia - Chile. 


\title{
Technological training in secondary-school music teachers. An intervention-based study on the integration of musical, technological and pedagogical contents at the University of Valencia
}

\begin{abstract}
This descriptive-exploratory work is part of a larger work carried out in Chile and Spain. Its objective has been to strengthen the technological training of future music teachers in secondary education through the design, implementation and evaluation of a subject in two consecutive cohorts (cohorts 2017 and 2018, n= 81). The design of the intervention took into account technological literacy components, and two learning strategies: projects $(A B P)$ and cooperative work. The novelty of the design consists in the adaptation of a theoretical framework for the integration of disciplinary-technological-pedagogical contents called TPACK that tries to improve the understanding of technology and its application in classroom teaching practice. The training products, the objects of the work projects, were didactic materials applicable in the classroom of the secondary school. The results of both cohorts suggest that the characterizing components of the intervention can help to plan more reflectively the use of music technology in the classroom. In addition, students claim to have improved their auto-perception of their technological skills in teaching situations and to have given meaning to technology in their profession.
\end{abstract}

\section{Key words}

Initial teacher training; music education; technological pedagogical content knowledge (TPACK); music technology; project-based-earning;

\section{Introducción}

Un uso efectivo de la tecnología de la información y comunicación (TIC) en los centros de educación secundaria pasa necesariamente por la formación inicial docente. En esta, se debe abordar espacios para que el profesorado en formación pueda asignar significados a su uso en los contextos escolares. Dichos espacios deben favorecer la interrelación con las otras materias del plan de estudios (Overbay, Patterson, Vasu y Grable, 2010), es decir, el profesorado ha de tener la capacidad de saber qué tecnología, cómo usarla y cuándo hacerlo para abordar las materias curriculares.

Las creencias, actitudes y confianza en las competencias y desempeño en el uso de la tecnología en el aula es un factor fundamental en la formación inicial del profesorado (Crawford, 2008; Voogt, Knezek y Somekh, 2008). Los resultados de investigación muestran resistencias hacia la tecnología en relación con la integración organizativa y curricular, la formación inicial tecnológica y la escasez u obsolescencia de recursos, entre otros factores (Boza, Tirado y Guzmán-Franco, 2010; Campos-Martínez, 2015; Crawford, 2008; Escudero, Martínez y Nieto, 2018; Voogt, Knezek y Somekh, 2008). 
Se ha sugerido que la capacidad de usar las TIC puede ser un predictor de su uso (Sang, Valcke, Braak y Tondeur, 2010), sin embargo, algunos planes de estudio no dan la suficiente importancia a este importante aspecto (Haning, 2016; Istenic, Coti, Solomonides y Volk, 2016). Así, algunas universidades dispusieron que las TIC deberían ser contenidos transversales de todo el currículum después de organizar los planes de estudio de acuerdo al Espacio Europeo de Educación Superior (Aguaded, 2009). De esto se podría deducir que no es necesaria una materia específica de formación tecnológica, pues las propias materias disciplinares o las troncales serían capaces de abordar dichos contenidos formativos.

Este estudio justifica con dos premisas la necesidad de abordar una alfabetización tecnológica específica, no transversal, en la formación inicial de profesorado. Por una parte, los y las jóvenes sobrevaloran su capacidad de leer y evaluar información en línea, seguramente debido a su auto-percepción positiva al participar en redes sociales y videojuegos (Larson, Forzani y Leu, 2018). El alumnado que ingresa en la universidad no es especialmente diestro en leer información compleja en línea, lo que tendría un efecto en el aprendizaje. Asimismo, encuentra difícil juzgar la exactitud, fiabilidad y sesgo de la información que encuentra en línea (Larson et al., 2018). Esto podría relativizar el concepto de nativos digitales de Prensky (2001), que ha sido contestado en otros trabajos (Ainley, 2018; Kirschner y De Bruyckere, 2017).

La literatura sobre formación tecnológica en la preparación inicial del profesorado es bastante amplia. Parece existir un cierto consenso sobre la función mediadora de las TIC entre las ciencias educativas, por una parte, y los problemas de aprendizaje, por otra (Osborne y Hennessy, 2003), aunque no es el remedio universal para los problemas pedagógicos que plantea la educación. El presente trabajo no parte del mito de la bondad de la tecnología educativa, sino de los procesos de uso y aprendizaje de la tecnología en un entramado holístico, constructivista y de interacción, con una comprensión del papel que el profesorado ha de ejercer en los centros educativos (Sancho, Bosco, Alonso y Sánchez, 2015). Desde esta premisa, se presenta el diseño de un módulo (Tecnología Musical) de una materia (Aprendizaje y Enseñanza de la Música), dentro del Máster de Profesorado de Educación Secundaria que oferta la U. de Valencia. Para ello, se ha adoptado un modelo pedagógico basado en tres elementos.

El primero adopta varios componentes de alfabetización tecnológica que proponen Mioduser, Nachmias y Forkosh-Baruch (2008) junto al constructo general de alfabetización o literacidad de Snow (2004).

El segundo es la estrategia de aprendizaje basado en proyectos cooperativos (ABP), una forma de enseñanza activa centrada en el alumnado caracterizada por la investigación constructiva, comunicación entre pares, objetivos predeterminados y trabajo reflexivo mediante actividades reales (Kokotsaki, Menzies y Wiggins, 2016). ABP ha dado resultados positivos en un buen número de experiencias en diferentes etapas educativas (Fernandes, Mesquita, Flores y Lima, 2014; Ljung-Djärf, Magnusson y Peterson, 2014; Mettas y Constantinou, 2008).

El tercer componente es el marco denominado Conocimiento del Contenido Tecnológico Pedagógico (Technological Pedagogical Content Knowledge, TPCK, también conocido como TPACK) (Mishra y Koehler, 2006; Mishra, Koehler y Henriksen, 2011). TPCK pretende facilitar la integración de diferentes contenidos en la formación inicial docente con el fin de construir un contexto mucho más sólido que la simple alfabetización tecnológica. TPCK tipifica los 
contenidos de cualquier materia en tecnológicos (TK), disciplinares (CK) y pedagógicos (PK). Sus intersecciones conforman: 1) el conocimiento pedagógico del contenido (PCK), relativo al proceso de enseñanza, es diferente para cada área de conocimiento y combina contenidos disciplinares y pedagógicos para desarrollar una mejor práctica docente. 2) el conocimiento tecnológico del contenido (TCK) aborda las tecnologías a usar en la enseñanza de una materia particular. 3) El contenido pedagógico-tecnológico (TPK) es necesario para conocer la pedagogía de los medios. 4) El conocimiento tecnológico-pedagógico del contenido (TPCK) es el necesario para integrar la tecnología en la práctica docente en cualquier materia (Koehler, Mishra y Cain 2013; TPACK, 2010) (figura 1). No obstante, TPCK no especifica cómo debería ser llevada a cabo la integración de contenidos, reconociendo explícitamente que pueden existir múltiples formas de abordarla (Harris, Mishra y Koehler, 2009; Mishra y Mehta, 2017).

\section{Figura 1.}

Componentes del modelo TPCK (TPCK-Technological, Pedagogical Content Knowledge, 2010).

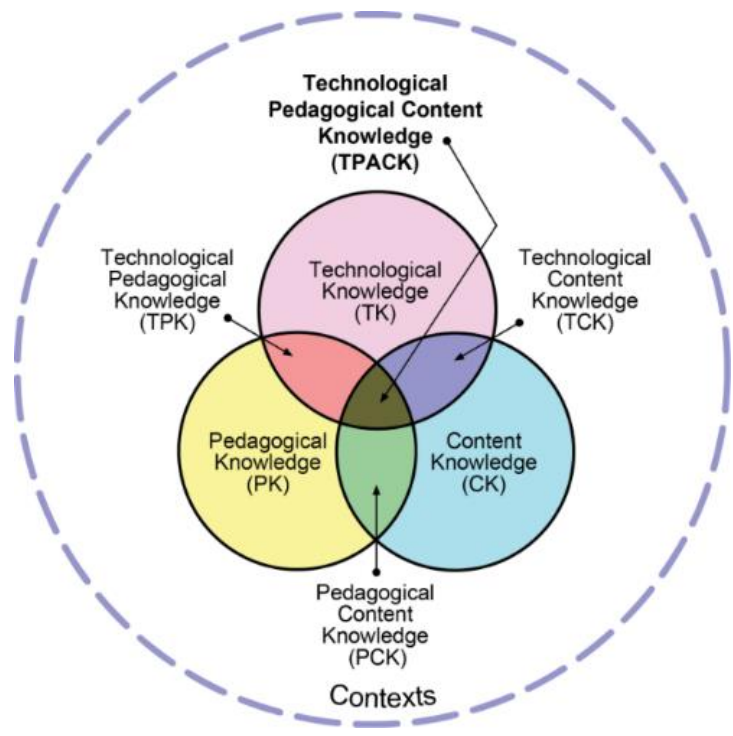

La importancia de la tecnología aplicada a la educación es parte de un cambio paradigmático que ha facilitado que el alumnado tenga más ocasiones de aprender mediante una variedad de situaciones, actividades y experiencias formativas (Cabero y Barroso 2018). El profesorado que ha integrado prácticas innovadoras utilizando tecnología ha verificado beneficios en la formación del alumnado (Murillo y Krichesky, 2015). Parece consecuente que en una sociedad cada vez más tecnificada, los centros educativos integren las TIC de manera pertinente en sus procesos formativos y didácticos (Kumar y Kumar, 2018; Pozo, López, Moreno y López, 2019). En este sentido, se ha evidencia la incidencia de factores inherentes a la práctica educativa en el nivel de competencia digital del profesorado (Pozo-Sánchez, LópezBelmonte, Fernández y López, 2020).

La intervención aquí presentada, inserta en la formación inicial del profesorado de educación secundaria, ha pretendido facilitar la alfabetización o literacidad tecnológica, fomentar el trabajo en equipo y, desde la perspectiva de la interacción simbólica, construir artefactos que 
ayuden a comprender los fenómenos que se conforman cuando se revisan simultáneamente contenidos disciplinares, didácticos y tecnológicos. Como investigación, pretende aportar datos de concreción al marco TPCK en la elaboración de intervenciones en educación musical.

\section{Contexto de la Intervención}

Esta es una investigación que parte de un proyecto de más largo alcance. En una anterior publicación (Tejada y Thayer, 2019a), se mostró resultados preliminares de una intervención similar a esta en una universidad chilena y otra publicación (Tejada y Thayer, 2019b) lo hizo en la formación de Maestro de Educación Primaria, especialidad Educación Musical, en la U. de Valencia.

La intervención de este estudio se realiza en la especialidad del Máster de Formación del Profesorado de Educación Secundaria de la U. de Valencia, un máster profesional de un año de duración. El módulo intervenido descrito aquí ha sido Tecnología Musical (24 horas presenciales), incluido en la materia Aprendizaje y Enseñanza de la Música, una asignatura obligatoria del máster en su itinerario de Música. El profesorado egresado de este máster está habilitado para impartir educación musical en centros públicos de educación secundaria.

El problema de investigación se dilucidó mediante una indagación libre en clase de las representaciones mentales del alumnado sobre el uso de la tecnología musical. Los resultados de observación mostraron: 1) conocimientos deficientes sobre uso de software y configuración de hardware con fines educativos; 2) reducidas experiencias previas con software y, en caso positivo, limitadas al uso de editores de partituras y editores de audio; 3 ) carencia de equipamientos informáticos adecuados en el centro donde reciben clase, así como la falta de una sala de grabación para trabajar con tecnología musical. Todo esto tiene relación con la docencia previa de tecnología en dicha materia: los objetivos, contenidos y actividades estaban centrados en la información descontextualizada de los procesos de enseñanza de hardware y software particulares, manteniendo escasa relación con contenidos musicales y pedagógicos. En resumen, no existía un programa centrado en el desarrollo de la competencia profesional docente sensible hacia las creencias del alumnado, ni articulación entre teoría y práctica, ni una aplicación directa al currículo escolar, ni una reflexión constante en la acción y sobre la acción (Tagle, 2011). Para dar alguna respuesta a este problema, se realiza el diseño de intervención presentado a continuación.

\section{Diseño de la Intervención}

La intervención concretó como modelo básico varios proyectos de trabajo (ABP) a realizar en pequeño grupo (aprendizaje colaborativo) integrando tres tipos de contenidos (marco TPCK). El objetivo didáctico de los proyectos fue la creación de materiales para la enseñanza de música instrumental en el aula de educación secundaria. Sigue a continuación el posicionamiento técnico-teórico del diseño.

\subsection{Modelo de alfabetización tecnológica}

Un elemento de este modelo parte de la definición de alfabetización como "social, con apoyo de la comunidad, culturalmente definida, variada y potencialmente transformadora" (Snow, 2004, p. 276). Otro elemento es la adopción de habilidades de alfabetización tecnológica de 
Mioduser et al. (2008): 1) Procesar información en múltiples modos de presentación. 2) Navegar por el espacio de información. 3) Comunicar éticamente la información a través de diferentes canales. 4) Decodificar, evaluar, usar o crear imágenes de diferentes tipos. Estas habilidades se desarrollan a través de la elaboración de proyectos.

\subsection{Interrelación de contenidos}

Se adopta el marco TPCK en la intervención. La siguiente figura muestra los contenidos integrados en cada proyecto y sus tipos.

\section{Figura 2.}

Esquema de contenidos de los proyectos de trabajo de la asignatura (TK: tecnológicos; CK: musicales; PCK: pedagógicos de música; PK: pedagógicos generales).

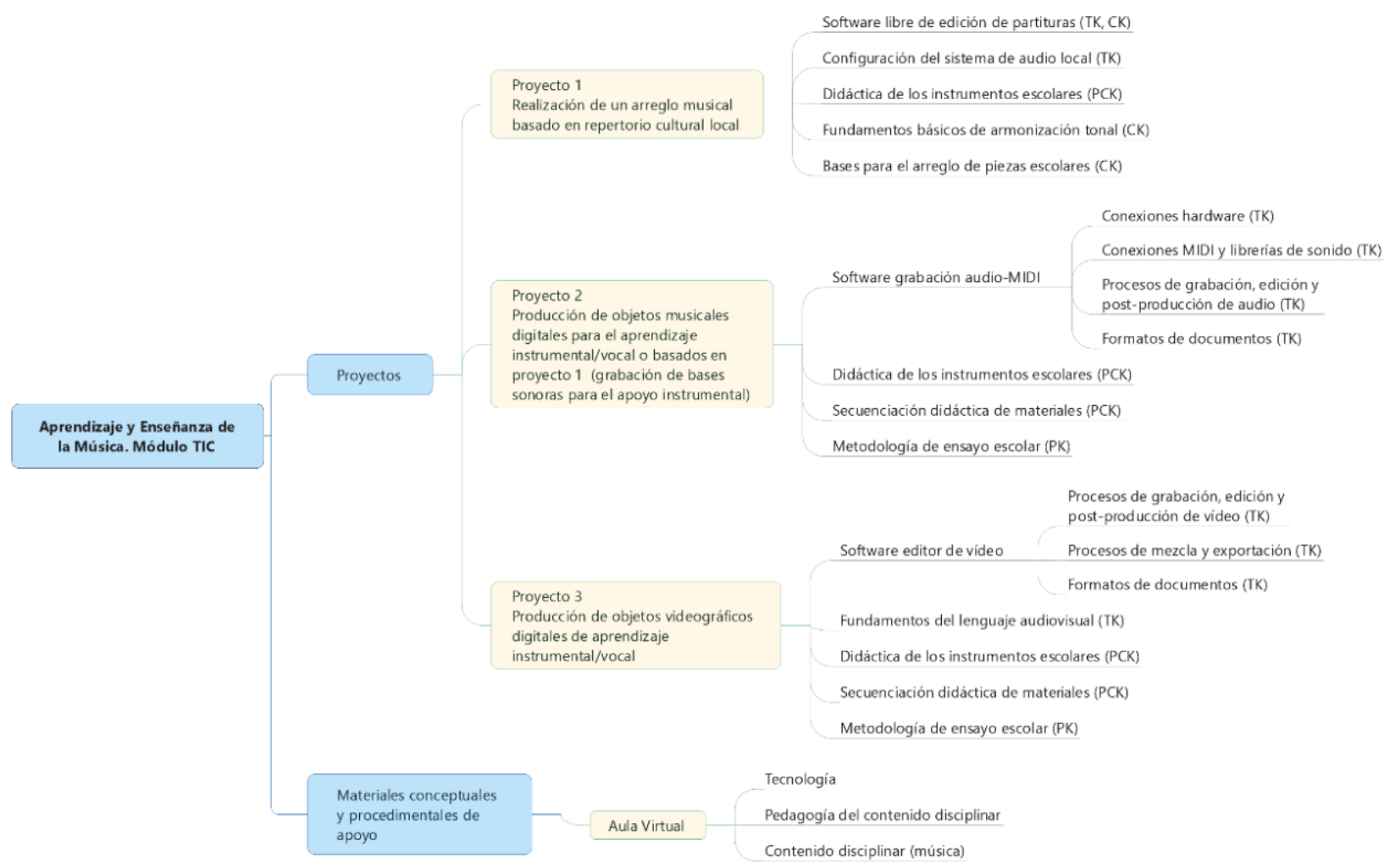

\subsection{Aprendizaje basado en proyectos}

ABP es el otro componente de esta intervención. Es definido como una forma de enseñanza activa centrada en el alumnado que se caracteriza por la investigación constructiva y social, comunicación inter-pares, objetivos predeterminados y trabajo reflexivo mediante actividades reales (Kokotsaki et al., 2016). La figura 2 detalla los proyectos cooperativos: tareas reales de elaboración materiales didácticos para la interpretación de piezas musicales con conjunto instrumental escolar susceptibles de ser usados en el aula de música de ESO. 
Los proyectos están relacionados y ordenados: dependen uno de otro y constituyen un sistema integral.

\subsection{Descripción de proyectos \\ 3.4.1 Proyecto uno: creación de un arreglo musical}

Consiste en elegir una canción popular de un repertorio cultural local y realizar un arreglo para cuatro o más instrumentos musicales escolares mediante software libre de edición de partituras. La Moodle de apoyo teórico del curso proporciona información declarativa y procedimental sobre los tres tipos de contenidos: 1) disciplinares (armonía elemental, complementariedad rítmica, creación de partes acompañantes); 2) tecnológicos (interfaz gráfica del software, configuración de audio, creación de particellas, exportación MIDI); y 3) pedagógicos disciplinares (resolución de potenciales problemas de aprendizaje instrumental del alumnado de ESO). El producto final es una serie de partituras (grupal e individuales) que son el punto de partida del proyecto dos.

\subsubsection{Proyecto dos: grabación multipista del arreglo musical}

En este proyecto se graba el arreglo realizado en el proyecto uno con instrumentos escolares o los propios del alumnado. Los productos finales de este proyecto son playbacks, es decir, bases musicales que sirven para apoyar la interpretación con instrumentos musicales escolares. Este proyecto se realiza mediante software libre para grabación multipista. La Moodle del curso proporciona vídeos que ejemplifican contenidos procedimentales con el software de grabación. Los contenidos pedagógicos de música tratan la secuenciación didáctica de los materiales grabados y el orden en que se expondrán en la clase de música. Así, el profesorado en formación comprende la lógica tanto de los materiales didácticos que crean como los pasos a seguir para usarlos en una situación hipotética de clase.

\subsubsection{Proyecto tres: Grabaciones de vídeo}

Consiste en grabar en vídeo de las interpretaciones instrumentales del proyecto 2 con los teléfonos personales del alumnado y la edición posterior con software libre. En la Moodle, hay información procedimental del editor de vídeo, así como contenidos pedagógicos sobre la secuencia didáctica a seguir en el aula. El resultado final de este proyecto es un material didáctico que se usará en el aula de ESO para facilitar la visualización y escucha de cada una de las partes instrumentales del arreglo.

\section{Método}

El presente trabajo sigue un enfoque mixto a partir de un diseño descriptivo-exploratorio, dada la ausencia de literatura de investigación en la aplicación del modelo TPCK y de la aproximación ABP en la formación inicial docente de profesorado de educación musical en educación secundaria (Máster de Profesorado de Educación Secundaria, especialidad Música, Universidad de Valencia). En este trabajo se presentan los resultados de dos intervenciones con sendas cohortes (2017-18 y 2018-19). La segunda tiene el objetivo de validar los resultados de la primera intervención. 


\subsection{Participantes y su caracterización [Nota 1]}

El alumnado de la cohorte 2017-18 ( $\mathrm{C} 1$, a partir de ahora) estuvo compuesto por 41 personas (19 mujeres y 22 hombres; $M=25,97$ años). El alumnado de la cohorte 2018-19 (C2), estuvo compuesto por 40 personas (21 mujeres y 19 hombres, $M=25,71$ años). La titulación mayoritaria de acceso al máster fue de Profesor Superior de Música ( $\left.C_{1}=29 ; C_{2}=24\right)$, la mayoría de especialidad instrumental.

Como parte del diseño de intervención y con el fin de recoger datos para construir el perfil de participante, se administró un cuestionario final (véase más adelante), con secciones que recogieron diferentes informaciones (véase sección instrumentos).

Declaran haber cursado música en educación primaria y secundaria, la mayoría en centros públicos. Valoran desigualmente la enseñanza musical recibida, de forma neutra en primaria y con sesgo negativo en secundaria.

Tabla 1.

Tipos de centro donde el alumnado cursó la materia de Música y la valoración que hicieron.

\begin{tabular}{llr|r|r} 
& & $C_{1}$ & $C_{2}$ & Total \\
\hline $\begin{array}{l}\text { Tipo de centro educativo } \\
\text { donde cursaron educación } \\
\text { primaria }\end{array}$ & Público & 19 & 18 & 37 \\
\cline { 2 - 5 } $\begin{array}{l}\text { Concertado o Privado } \\
\text { donde centro educativo } \\
\text { secursaron educación }\end{array}$ & Público & 14 & 10 & 24 \\
\cline { 2 - 5 } & Concertado o Privado & 10 & 25 & 45 \\
\hline $\begin{array}{l}\text { Valoración de materia en } \\
\text { ed. primaria } \\
\text { (escala 5 puntos) }\end{array}$ & Media & 3,15 & 3,23 & 3,19 \\
\hline $\begin{array}{llll}\text { Valoración de materia en } \\
\text { ed. secundaria } \\
\text { (escala 5 puntos) }\end{array}$ & SD & 1,034 &, 762 &, 906 \\
\cline { 2 - 5 } & SD & 2,68 & 2,48 & 2,58 \\
\hline
\end{tabular}

Durante su escolarización en educación primaria y secundaria, los participantes declaran haber cursado simultáneamente formación musical reglada. La experiencia previa con tecnología es desigual en ambas cohortes. Usan software de edición de partituras con relativa frecuencia y software de edición de audio.

En relación al hardware, los aparatos que más reconocen son los grabadores y reproductores de audio, seguido de altavoces activos-pasivos y micrófonos dinámicos, quizá porque los hayan visto durante sus grabaciones en estudios de sonido, en su mayoría profesionales (ítems 22 y 23 de la encuesta inicial). Tienen una auto-percepción moderadamente positiva de sus habilidades tecnológicas. El software que más utilizan son los editores de vídeo, los grabadores y editores de audio, quizá porque hayan trabajado con ellos durante sus estudios musicales.

\subsection{Instrumentos}

Se usaron tres instrumentos de recogida de datos: 1) tres rúbricas de evaluación de producto (cuantitativas; una rúbrica por proyecto); 2) un informe de auto-evaluación de los alumnos para evaluar la intervención y recoger percepciones del alumnado (cualitativo); y 3) un cuestionario posterior a la intervención (items abiertos y cerrados) que recogió información sobre aspectos personales, percepciones sobre la calidad de la educación musical recibida en 
la educación obligatoria, estudios musicales previos al máster, la autopercepción sobre sus competencias TIC y la autopercepción de su capacidad para el uso de la tecnología en procesos de enseñanza musical.

Cada rúbrica de proyecto tuvo un porcentaje diferenciado en la calificación final, dependiendo de la complejidad y del tiempo necesario para completar el proyecto. Cada rúbrica tuvo una validación a partir de las opiniones de dos expertos sobre la adecuación del proyecto a los objetivos y el peso del mismo en la calificación (kappa de Cohen k=.94 proyecto uno; $\mathrm{k}=.92$ proyecto dos; $\mathrm{k}=.95$ proyecto tres).

Las rúbricas para cada proyecto fueron:

Proyecto 1 (20\% de la calificación final) [Nota 2]

- Adecuación a las habilidades de alumnado de secundaria (PCK).

- Inclusión de ostinati [Nota 3] como recurso didáctico (PCK).

- Coherencia armónica (CK).

- Adecuación para instrumentos escolares (PK, PCK).

- Adecuación y coherencia rítmica (CK).

- Corrección técnica de la partitura creada (TK, CK).

Proyecto 2 (35\% de la calificación final)

Este proyecto (grabación con instrumentos escolares) tuvo una clara intención didáctica: la elaboración de fondos sonoros (playbacks) sobre los cuales los escolares pueden tocar sus instrumentos.

Los criterios de la rúbrica fueron:

- Afinación en la interpretación de cada instrumento o voz (CK)

- Exactitud rítmica y sincronización de la grabación entre los diferentes instrumentos, evitando la cuantización excesiva de las muestras grabadas (TK, CK).

- Expresividad musical (uso de agógica y dinámicas)(CK).

- Duración mínima adecuada al tiempo de atención del alumnado de ESO (PK).

- Producción de minus-ones (karaokes) adecuados a una secuencia didáctica en aula (PK, PCK).

- Uso de parámetros: panorama (balance de planos sonoros), ecualización (compensación acústica) y volumen (TK).

- Uso de efectos (reverberación, delay, compresor y otros)(TK).

- Inclusión de pista de datos MIDI y activación con instrumentos digitales (TK).

Proyecto 3 (35\% de la calificación final)

Este proyecto (grabación de vídeos de cada instrumento) tiene como objetivo mostrar al alumnado de ESO cómo se interpreta cada parte y evitar el uso de notación occidental. Los criterios de evaluación de este proyecto fueron:

- Correcto encuadre y tamaño (el intérprete o sus manos están centrados en la imagen; no se debe hacer una toma con un instrumento alejado: debe poder percibirse correctamente las acciones en el instrumento) (PK, PCK, TK).

- Visión subjetiva del instrumento "en espejo" para que el alumnado pueda imitar los movimientos (PK, PCK, TK)

- Calidad de sonido aceptable (dinámica, espectro tímbrico) (CK, TK). 
La auto-evaluación consistió en un informe abierto de cada participante. En la descripción, los investigadores sugirieron categorías generales para facilitar las respuestas de forma estructurada.

\subsection{Procedimiento}

El módulo TM tiene asignadas 24 horas lectivas ( 2 sesiones semanales de 2 horas), además de las sesiones de proyectos. Se programó las sesiones prácticas de proyectos. Se informó al alumnado matriculado de los términos de la investigación y se procedió a firmar el correspondiente consentimiento informado. Después, se formaron grupos para los proyectos. Asimismo, se publicaron los materiales de apoyo y el contenido y actividades de cada proyecto en la Moodle del curso.

En las clases presenciales, cada grupo trabajó su primer proyecto en un aula general de informática. Comenzó con la selección de una melodía de la cultura local. Después, transcribieron la canción seleccionada mediante software libre de edición de partituras y comenzaron a arreglarla para un mínimo de 4 instrumentos escolares a partir de decisiones musicales guiadas por criterios pedagógicos.

Los proyectos 2 y 3 fueron grabados por cada grupo en dos aulas de la facultad con equipamiento de grabación (ordenador, interfaz, auriculares y micrófono) y fue realizado en horario no lectivo. Las sesiones del proyecto 2 fueron tutorizadas por el profesor de la asignatura, debido a la especificidad de la información a entregar en la primera sesión de grabación. Se realizaron actividades con el software de grabación y directrices de utilización en aula de los materiales a crear en el proyecto, lo que supuso abordar también contenidos tecnológicos específicos y pedagógicos. El proyecto 3 consistió en la toma inicial de vídeo durante la grabación de cada instrumento y luego la edición mediante software libre.

\section{Análisis de datos}

En primer lugar, se presenta la evaluación de los productos y después el análisis de las percepciones del alumnado en sus autoevaluaciones.

\subsection{Resultados de productos}

La rúbrica cerrada de cada proyecto incluyó criterios en relación al contenido disciplinar, pedagógico y tecnológico. El contraste de medias muestra diferencias estadísticamente significativas entre $\mathrm{C}_{1}$ y $\mathrm{C}_{2}$ en el proyecto 1 (a favor de $\mathrm{C}_{2}$ ) y en el proyecto 2 (a favor de $\mathrm{C}_{1}$ ) con un tamaño de efecto considerable (tabla 2 y figura 3 ). No obstante, la diferencia no obedece a ningún cambio en el diseño de intervención, pues fue igual para ambas cohortes. Esto fue debido a que se pretendía validar la intervención, no contrastar resultados de cohortes en un tipo de investigación-acción. Por ello, la importancia de estos datos no radica en la diferencia, sino en las puntuaciones absolutas de los productos, en un rango medio muy aceptable y, sobre todo, la relación que mantienen estas puntuaciones con las percepciones de los participantes. 
Tabla 2.

Contraste de medias de puntuación de los proyectos.

\begin{tabular}{|c|c|c|c|c|c|c|}
\hline & Cohorte & $\mathrm{N}$ & Media & DT & Sig. & $\begin{array}{l}\text { T. efecto } \\
\text { (D Cohen) }\end{array}$ \\
\hline \multirow[t]{2}{*}{ Proyecto1 } & $\mathrm{C}_{1}$ & 41 & 6,610 & 1,9446 & \multirow[b]{2}{*}{,017 } & \multirow[b]{2}{*}{,- 8402} \\
\hline & $\mathrm{C} 2$ & 40 & 7,450 & 1,0051 & & \\
\hline \multirow[t]{2}{*}{ Proyecto2 } & $\mathrm{C}_{1}$ & 41 & 6,854 & 1,9080 & \multirow[b]{2}{*}{,017 } & \multirow[b]{2}{*}{,9287 } \\
\hline & $\mathrm{C} 2$ & 40 & 5,925 & 1,4787 & & \\
\hline \multirow[t]{2}{*}{ Proyecto3 } & $\mathrm{C}_{1}$ & 41 & 8,817 & 8348 & \multirow[b]{2}{*}{,094 } & \multirow[b]{2}{*}{ 2671 } \\
\hline & $\mathrm{C} 2$ & 40 & 8,550 &, 5524 & & \\
\hline
\end{tabular}

Figura 3.

Resultados evaluaciones de proyectos por cohorte

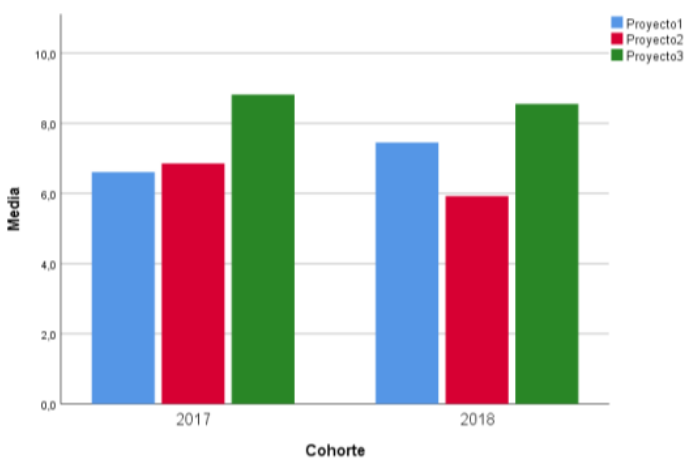

\subsection{Percepciones del alumnado}

Cada persona realizó un documento abierto de auto-evaluación. A título de sugerencia, se proporcionaron cinco pautas orientadoras generales (no preguntas directas) que trataron de estructurar la información de los participantes de acuerdo a las categorías de información pretendidas:

- "Puede indicar sus ideas sobre esta asignatura: utilidad, adecuación al nivel universitario que cursa u otras ideas generales que quiera mencionar.

- Puede indicar qué ha aprendido y qué no ha aprendido en esta materia

- Puede indicar sus expectativas sobre esta asignatura ¿qué se ha cumplido y qué no se ha cumplido?

- Puede indicar opiniones concretas tiene de esta materia en relación a los proyectos, trabajo colaborativo, organización, infraestructuras, evaluación, repertorio, dificultades, logística, trabajo autónomo...

- Puede indicar una puntuación razonada que se daría por el trabajo realizado considerando su asistencia, dedicación y resultados"

Se realizó un análisis de contenido en función de las categorías deducidas en este instrumento y posteriormente, mediante un proceso recursivo y relacional, se agruparon los 
códigos, induciendo tres categorías: 1) aspectos académicos; 2) aspectos negativos; 3) aspectos positivos de la asignatura (figura 4 ).

\section{Figura 4 .}

Mapa de categorías extraídas de las auto-evaluaciones. Entre paréntesis, número de unidades significativas (US) contenidas en el código de análisis.
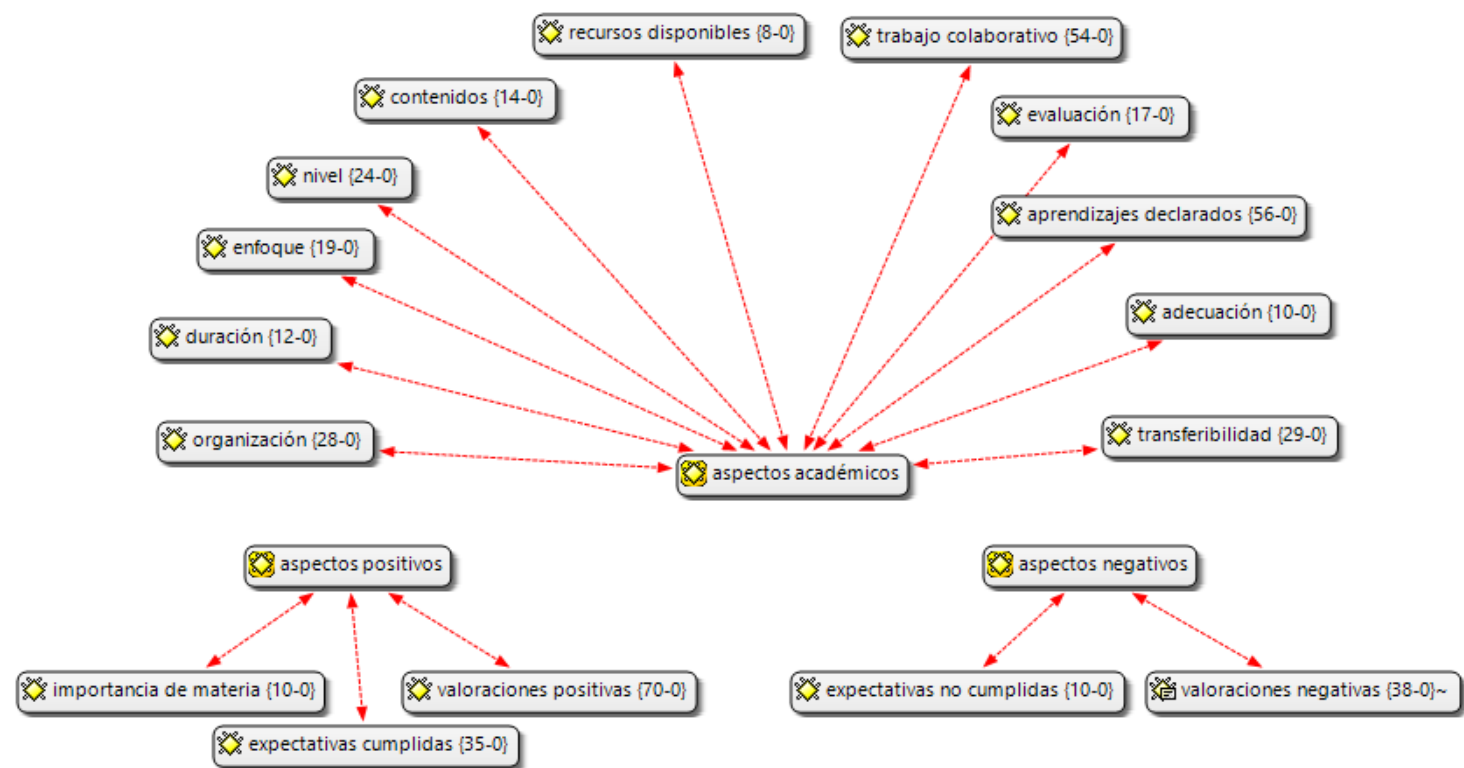

La categoría aspectos académicos incluyó los códigos organización, duración, enfoque, nivel, contenidos, recursos disponibles, evaluación, trabajo colaborativo, evaluación, aprendizajes, adecuación, transferibilidad.

Las opiniones sobre organización del módulo ( 28 unidades de significado, entre paréntesis en la fig. 4) son totalmente positivas, con manifestaciones como: "muy bien organizada", "bien estructurada", "buen sistema organizativo", "muy correcta", "me ha parecido uno de los aspectos más positivos".

La duración del módulo de materia fue valorada de forma unánime como de corta duración, poco extensa o insuficiente, expresando de forma unánime el contraste entre la importancia asignada a la materia por el alumnado (o bien su dificultad; véase más abajo) y la duración del módulo.

El código enfoque de las TIC como: 1) herramienta docente; 2) integradora de contenidos; 3 ) estrategia de aprendizaje basada en proyectos; 4) estrategia de trabajo grupal, ha tenido gran concurrencia de opiniones positivas, por ejemplo: "hemos aprendido a hacer por nosotros mismos recursos tecnológicos para implementar en el aula" ( $(\mathrm{C}, 4: 118)$. "ha estado enfocada de forma práctica, lo que hace que tengamos los contenidos más asumidos; [...] cabe resaltar la libertad en cuanto a la creatividad del alumnado, ya que no es algo que, por norma general, se potencie en las aulas" $(C 2,5: 136)$. 
El alumnado opina muy diferentemente respecto al nivel (dificultad) de la materia. Algunas personas creen que el nivel ha sido básico porque la duración del módulo no se prestaba a más o porque su nivel de conocimientos era mayor: "La adecuación y nivel han sido básicos y ajustados" $(\mathrm{C} 2,5: 67)$. Para otras personas, el nivel fue excesivo: "El nivel [...] es elevado, pues no tenía muchos conocimientos digitales, pero, cabe decir que la asignatura comienza desde conceptos básicos y va avanzando hacia la utilización de los programas (uso explicado de forma bastante individualizada)" ( $(1,4: 155)$. Mientras, otras creen que el nivel ha sido óptimo o bien adecuado: "En cuanto a la adecuación y el nivel lo considero óptimo. Los programas pueden ser utilizados para trabajar en el aula de secundaria como en la de primaria, y preparar las clases, además de poder realizar ejercicios diferentes". (C2, 5:102).

Los contenidos son valorados casi unánimemente como adecuados: “muy bien adecuados a los intereses y perfiles de los alumnos, pues se nos ha abierto un horizonte a la hora de tener recursos dentro de un aula para poder trabajar" (C2; 5:163). Varias participantes opinan también sobre lo acertado de trabajar música de la cultura propia.

En el código trabajo colaborativo, se recogió más percepciones positivas que negativas: "se complementan conocimientos y afloran puntos de vista que nos hacen crecer como personas" ( 1 1,4:150), "nos ha permitido... no solo aprender de la materia en sí, sino también de los compañeros" ( $(1,4: 187)$. Como percepciones negativas, se manifiesta que el trabajo en grupo es más lento y cuesta más de organizar ( $\left.C_{1,4: 23 ;} C_{2}, 5: 135\right)$, que "no es fácil poner a trabajar al grupo" ( $(1,4: 167)$. Una de las críticas al trabajo colaborativo hace mención a un problema habitual: los miembros del grupo conciben el trabajo colaborativo como un reparto de tareas individuales, sin mención a las decisiones conjuntas sobre el producto final, lo cual lo convierte trabajo cooperativo.

El otro gran problema es la disponibilidad a trabajar de forma equitativa por los miembros del grupo: "la organización del grupo ha sido pésima, siempre nos hacemos cargo de los trabajos más costosos los mismos y siempre acabamos haciendo más faena que los demás" C1, 4:170)." Este fenómeno ha sido tipificado en otros trabajos a los que se remite al lector (Buchs, Filippou, Pulfrey y Volpé, 2017; Gundara y Sharma, 2013; Johnson y Johnson, 2014).

El código aprendizajes realizados es rico en resultados positivos e incluye muchas declaraciones que destacan la relación entre los aprendizajes y su aplicabilidad en aula: "El hecho de enfocar los procesos técnicos a la creación de recursos y su evidente adaptación a un determinado nivel, además del proceso de grabación propiamente dicho, sí que ha significado para mí un avance y un aprendizaje más que significativo de cara a la puesta en práctica de la formación que estamos adquiriendo" (C1, 4:205).

No obstante, hay personas que mencionan haber realizado aprendizajes tecnológicos sin declarar su aplicación en aula. Quizá esto se debe a que estas personas se perciben más como músicos que como profesores. No hay que olvidar que la mayoría del alumnado son instrumentistas egresados de conservatorios y que su vocación es la interpretación: "Ha sido interesante realizar grabaciones con instrumentos, ya que la acústica instrumental me gusta y me parece muy interesante la colocación de micrófonos para diferentes instrumentos y las posibilidades sonoras de estos" $(C 2,5: 8)$.

La mayoría de participantes ha valorado como correcto, adecuado, organizado... el sistema de evaluación y los porcentajes de cada proyecto: "Este es un módulo donde más que la teoría importa la adquisición de las competencias en el manejo de los programas propuestos. En mi 
opinión, la única manera de adquirir estas competencias es la actual organización del trabajo por proyectos y en grupos. Por lo tanto, tiene sentido que la evaluación sea principalmente la de dichos proyectos" ( $(1,4: 33)$.

Las opiniones sobre los aprendizajes realizados se triangulan bien con las opiniones vertidas sobre expectativas cumplidas, que dieron un resultado sorprendente: casi todas las personas manifestaron que la asignatura había cumplido sobradamente sus expectativas, expresándose con términos dentro de un campo semántico positivo ("sorprendente", "gratificante", "motivadora”, "libertad", “autonomía”, "satisfacción”, "acierto” y otras semejantes). Las expectativas no cumplidas arrojaron luz sobre los deseos del alumnado, exponiendo su deseo de haber aprendido a usar otro tipo de herramientas con más profundidad, como los editores de vídeo. Otra persona declaró que esperaba más de la asignatura en cuanto al nivel de profundización, aunque también menciona que su opinión no tuvo en cuenta el nivel medio de la clase en relación a la tecnología o la corta duración del módulo.

El código transferibilidad de aprendizajes agrupó las percepciones del alumnado respecto a las posibilidades de transferencia al aula de los aprendizajes realizados. En sus opiniones, el módulo ofreció vías para configurar un imaginario del uso de la tecnología desde una mirada pedagógica. Las opiniones son muy convergentes, por ejemplo: "es muy efectiva y muy pedagógica su aplicación en aula" ( $C 2,4: 58)$, "en el aula de música, es un recurso vital” ( $C 2$, 4:59). "La aplicación de [los programas trabajados] en el aula de secundaria es adecuada a todos los ciclos" ( $\left.\mathrm{C}_{1}, 5: 123\right)$. "Es un recurso muy útil [...] haciendo que el aprendizaje sea mucho más fácil para nuestro alumnado; es por esto que estoy segura de que lo voy a aplicar en un futuro en mis clases" ( $C 2,4: 224)$. "Cuando se planteó los diferentes proyectos que debían realizarse, fue una grata sorpresa, pues me parecieron totalmente interesantes y aplicables al aula de secundaria "( $C 2,4: 129)$.

En estas opiniones sobre la aplicación de la tecnología, subyace la idea de competencia en su aplicación, de autoconfianza, lo que constituye un indicador indirecto de la transferibilidad potencial de los aprendizajes realizados. El alumnado percibe que la tecnología podría facilitar la elaboración de materiales para el aula: "aplicaciones muy útiles a la hora de crear materiales para el aula" (C2, 5:130), "realmente ha sido de gran utilidad de cara a nuestro futuro como profesores tener herramientas y recursos para trabajar con nuestro alumnado y poder así hacer cosas más interesantes y poder preparar materiales adecuados al alumnado de cada curso" ( $\mathrm{C} 1,5: 66)$, "creo que para el alumnado es muy positivo la utilización de recursos tecnológicos en el aula $\mathrm{y}$, aunque sea un trabajo laborioso por parte del profesorado, considero muy efectiva y muy pedagógica su aplicación en el aula" ( $C 2,4: 58)$.

Estos datos también pueden interpretarse como indicios de la auto-confianza en sus habilidades y competencias en relación al uso de la tecnología. A este respecto, el cuestionario final (instrumento 3) incluyó varios ítems sobre auto-percepción en relación al uso de tecnología musical en aula. Los resultados son auto-explicativos (tabla 3). 


\section{Tabla 3.}

Respuestas a los ítems 24 y 25 de la encuesta

\begin{tabular}{|c|c|c|c|c|}
\hline & & $\mathrm{C}_{1}$ & $\mathrm{C} 2$ & Total \\
\hline \multirow[b]{2}{*}{$\begin{array}{l}24 \text { ¿Cree sentirse capacitado para } \\
\text { usar software y aparatos de } \\
\text { tecnología musical para enseñar } \\
\text { música en su futuro trabajo como } \\
\text { docente? }\end{array}$} & No & 9 & 4 & 13 \\
\hline & $\mathrm{Si}$ & 24 & 27 & 51 \\
\hline \multirow[b]{2}{*}{$\begin{array}{l}25 \text { ¿Usted aplicaría diversas } \\
\text { herramientas de tecnología } \\
\text { digital, tales como } \\
\text { secuenciadores, sistemas de } \\
\text { grabación digital, adiestradores } \\
\text { auditivos y editores de sonido } \\
\text { para crear música para sus clases } \\
\text { de educación musical? }\end{array}$} & No & 3 & 1 & 4 \\
\hline & $\mathrm{Si}$ & 30 & 30 & 60 \\
\hline
\end{tabular}

El resultado concluyente fue proporcionado por el ítem 26, que preguntaba si la tecnología musical tiene algún papel en los procesos de enseñanza y aprendizaje de la música: todo el alumnado respondió afirmativamente. El ítem 27 de la encuesta (abierto) indagó sobre las razones de estas opiniones. Con los datos obtenidos, se realizó un análisis de contenido de tipo inductivo con el software Atlas.ti y se elaboró el mapa semántico de la figura 5.

\section{Figura 5.}

Mapa semántico de los motivos por lo que los participantes creen que la tecnología tiene un papel en los procesos de educación musical (entre paréntesis, número de unidades y número de relaciones del código).

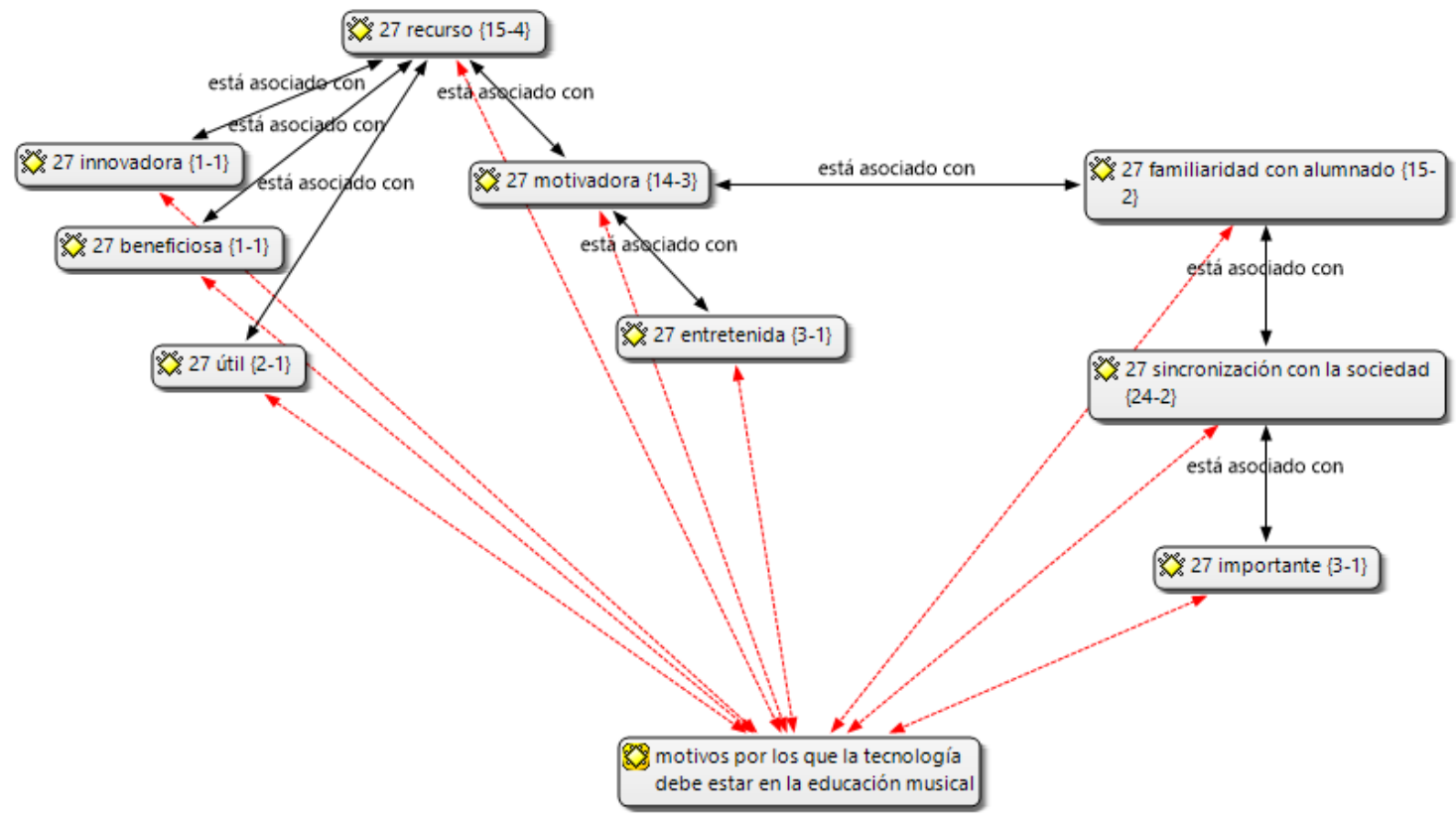


En cuanto a las sugerencias de los participantes, la mayoría estuvieron relacionadas con prolongar la duración del módulo, lo que también proporciona un indicador de la motivación de los participantes.

\section{Conclusiones}

El módulo de la asignatura ha tenido muy buena aceptación por el alumnado, de acuerdo a las manifestaciones recogidas en la categoría aspectos académicos. El enfoque de la asignatura ha sido explícitamente reconocido como una integración de contenidos; el modelo formativo se ha valorado como muy útil en la transferibilidad de los aprendizajes al desempeño docente en el aula; el nivel de materia ha sido reconocido como adecuado para el nivel de conocimientos previos; la organización ha sido correcta y bien evaluada; los contenidos han sido adecuados y consistentes con los objetivos. Esta buena aceptación ha sido extensiva a la metodología, la evaluación y al sistema de trabajo por proyectos, que configuran modos para elaborar materiales didácticos. Más allá de estos resultados que validan la intervención, la integración de contenidos ha mejorado la autoconfianza en las propias habilidades docentes para trabajar con tecnología, lo que quizá pueda inducir a los estudiantes a reflexionar sobre la acción didáctica e investigar nuevas formas de uso de la tecnología en las materias curriculares. En relación a la discusión con el marco teórico, se induce que: 1) El alumnado opina muy positivamente sobre el trabajo colaborativo y el aprendizaje a partir de proyectos reales. Esto confirma los hallazgos sobre aprendizaje basado en proyectos (Kokotsaki et al. 2016). 2) La mayoría de personas perciben muy positivamente la relación existente entre los contenidos musicales, tecnológicos y pedagógicos, es decir, el marco TPCK, lo que confirma los trabajos teóricos publicados (Koehler et al. 2013; Mishra y Koehler, 2006; Mishra et al. 2011) y también los trabajos empíricos (Baran et al., 2011; Chai et al., 2010).

No obstante, las personas participantes revelan también algunos problemas que necesitan ser indagados en estudios posteriores. Por ejemplo, los problemas relacionados con el trabajo colaborativo, que se convirtió en algunos casos en una simple cooperación inter pares y que han sido reportados por Gundara y Sharma, (2013) o Johnson y Johnson (2014), entre otros. Este es un elemento importante en la formación de los futuros docentes, pues es necesario para el desempeño profesional colegiado. La pregunta a responder debería ser ¿Cómo se podría desarrollar estrategias para un trabajo colaborativo efectivo y no meramente trabajo cooperativo?

El valor de esta investigación ha sido proponer una metodología de integración de contenidos tecnológicos, disciplinares y pedagógicos en la formación pedagógica del profesorado de música que concreta cierta ambigüedad del marco teórico TPACK en el área. Además, la intervención introduce elementos de interacción que merecen estudio posterior, como ha sido el aprendizaje basado en proyectos y el trabajo colaborativo. Esta propuesta ha intentado aportar una nueva perspectiva al enfoque tecnológico predominante que ha caracterizado la inclusión de los contenidos de tecnología musical en muchas carreras de formación musical del profesorado (Savage 2010; Tejada, 2004). Por otra parte, abre una alternativa al profesorado de educación musical que tiene nociones pedagógicas, pero carece en general de competencias digitales. 
No obstante, este estudio requiere de investigación ulterior que permita validar sus resultados. Esto permitirá llegar a una concreción operativa del modelo TPCK en educación musical, todavía por realizar. La adopción de estrategias de integración de contenidos en la formación inicial del profesorado de música de educación secundaria podría facilitar el pensamiento crítico y reflexivo y, con ello, la operativización de la tecnología en los procesos de aprendizaje. Asimismo, podría orientar y guiar a las administraciones educativas en la implementación de nuevas líneas estratégicas en la formación del profesorado en relación al uso de las TIC en diferentes contextos educativos. De esta manera, se podría responder de forma más coherente e innovadora a una variedad enorme de estilos y necesidades de los estudiantes desde estrategias innovadoras creativas, constructivas, implicativas y polivalentes orientadas al aprendizaje.

\section{Notas}

[1] Para que el lector no conocedor del sistema educativo español pueda comprender el perfil del alumnado participante, es necesario explicar que las Enseñanzas Superiores de Música (equivalente a un grado, pero sin serlo explícitamente) se imparten en los conservatorios superiores de España, que tienen un status académico diferente a las universidades. La titulación de egreso de estos conservatorios es la mayoritaria de acceso al máster de este trabajo. La titulación aquí abordada es un máster oficial de tipo profesional de un año de duración impartido en las universidades españolas que habilita a impartir educación musical en educación secundaria en centros públicos españoles. Este máster es obligatorio para acceder a un puesto de trabajo en un centro educativo público, pero no se exige para trabajar en centros privados.

[2] Tipos de contenidos: TK: tecnológicos; CK: musicales; PCK: pedagógicos de música; PK: pedagógicos generales.

[3] El ostinato es una breve agrupación de sonidos que se repiten cíclicamente en su ritmo, variando su altura en función de la armonización. Se suele utilizar para memorizar partes extensas sin necesidad de usar partituras y evitar así una exposición prematura a las complejidades de la notación musical occidental.

\section{Agradecimientos}

Este trabajo ha sido financiado por el Ministerio de Educación de Chile (Programa de Mejora Institucional, código PMI-EXA/PNII/05/2017) y el Ministerio de Ciencia e Innovación de España (Plan Nacional de i+d+i 2019, código PID2019-105762GB-Ioo) en cofinanciación con fondos FEDER.

\section{Referencias}

Aguaded, I. (2009). Miopía en los nuevos planes de formación de maestros en España ¿docentes analógicos o digitales? Comunicar, 33, XVII, 7-8. doi: 10.3916/c33-2009-00001.

Ainley, J. (2018). Students and Their Computer Literacy: Evidence and Curriculum Implications. En J. Voogt, G.Knezek, R. Christensen y Kwok-Wing Lai (eds.) Second Handbook of Information Technology in Primary and Secondary Education. (p. 70-88). Cham: Springer International Publishing.

Baran, E., Chuang, H. y Thompson, A. (2011). TPACK: an emerging research and development tool for teacher educators. TOJET: The Turkish Online Journal of Educational 
Technology, 10, 4, 370-377. Recuperado de: https://files.eric.ed.gov/fulltext/EJ946646.pdf

Boza, A., Tirado, R. y Guzmán-Franco, M. (2010). Creencias del profesorado sobre el significado de la tecnología en la enseñanza: influencia para su inserción en los centros docentes andaluces. RELIEVE, 16, 1. 1-24. doi:10.7203/relieve.16.1.4152

Buchs, C., Filippou, D., Pulfrey, C. y Volpé, Y. (2017). Challenges for cooperative learning implementation: reports from elementary school teachers. Journal of Education for Teaching, 43(3), 296-306. doi:10.1080/02607476.2017.1321673

Cabero, J., y Barroso, J. (2018). Los escenarios tecnológicos en Realidad Aumentada (RA): posibilidades educativas en estudios universitarios. Aula Abierta, 47(3), 327-336. doi: 10.17811/rifie.47-3.2018.327-336

Campos-Martínez, J. A. (2015). Lo cotidiano (entre usos y resistencias) de las TIC en un aula de la educación secundaria obligatoria. Apuntes etnográficos. Antropología Experimental, (15). doi:10.17561/rae.voi15.2633

Chai, C. S., Koh, J. H. L., y Tsai, C.-C. (2010). Facilitating Preservice Teachers' Development of Technological, Pedagogical, and Content Knowledge (TPACK). Educational Technology \& Society, 13 (4), 63-73. Recuperado de: https://pdfs.semanticscholar.org/dcb3/60e8cb725db4e36b692be410224ddaf74db1 .pdf

Crawford, R. (2008). Are Resources Solely to Be Blamed? The Current Situation on Music Education Facilities, Computer and Music Technology Resources in Victoria. Australian Journal of Music Education, 1. 44-55. Recuperado de: https://search.informit.com.au/documentSummary; dn=517151838294372; res=IELH SS

Escudero, J.M., Martínez, B., y Nieto, J.M. (2018). Las TIC en la formación continua del profesorado en el contexto español. Revista Educación, 382, pp. 57-80. doi: 0.4438/1988-592X-RE-2018-382-392

Fernandes, S., Mesquita, M., Flores, M.A. y Lima, R.M. (2014) Engaging students in learning: findings from a study of project-led education. European Journal of Engineering Education, 39:1, 55-67, doi: 10.1080/03043797.2013.833170.

Gundara, J. S., y Sharma, N. (2013). Some issues for cooperative learning and intercultural education. Intercultural Education, 24(3), 237-250. doi:10.1080/14675986.2013.797202

Haning, M. (2016). Are They Ready to Teach With Technology? An Investigation of Technology Instruction in Music Teacher Education Programs. Journal of Music Teacher Education, 25(3). 78-90. doi: 10.1177/1057083715577696

Harris, J., Mishra, P., y Koehler, M. (2009). Teachers' technological pedagogical content knowledge and learning activity types: curriculum-based technology integration reframed. Journal of Research on Technology in Education, 41(4), 393-416. doi: 10.1080/15391523.2009.10782536

Istenic, A., Cotic, M., Solomonides, I. y Volk, M. (2016). Engaging preservice primary and preprimary school teachers in digital storytelling for the teaching and learning of mathematics. British Journal of Educational Technology, 47(1), 29-50. doi: $10.1177 / 1365480216659733$ 
Johnson, D., y Johnson, R. (2014). Cooperative Learning in 21st Century. Anales de Psicología / Annals of Psychology, 30(3), 841-851. doi:10.6018/analesps.30.3.201241

Kirschner, P. A., y De Bruyckere, P. (2017). The myths of the digital native and the multitasker. Teaching and Teacher Education, 67, 135-142. Doi: 10.1016/j.tate.2017.06.001

Koehler, M., Mishra, P. y Cain, W. (2013). What is technological pedagogical content Knowledge (TPACK)? Journal of Education, 193, 3, 13-19. Doi: 10.1177/002205741319300303

Kokotsaki, D., Menzies, V. y Wiggins, A. (2016). Project-based learning: A review of the literature. Improving Schools, 19 (3). 267-277. doi: 10.1177/1365480216659733

Kumar, A. y Kumar, G. (2018). The role of ICT in higher education for the 21st century: ICT as a change agent for education. Multidisciplinary Higher Education, Research, Dynamics \& Concepts, 1(1), 76-83. Recuperado de: https://bit.ly/2NU3HV6

Larson, L., Forzani, E. y Leu, D.J. (2018). New Literacies: Curricular Implications. En J. Voogt, G.Knezek, R. Christensen y Kwok-Wing Lai (eds.) Second Handbook of Information Technology in Primary and Secondary Education. (pp. 38-52). Chan: Springer International Publishing.

Ljung-Djärf, A., Magnusson, A. y Peterson, S. (2014). From doing to learning: Changed focus during a preschool learning study project on organic decomposition. International Journal of Science Education, 36, 656-676. doi: 10.1080/09500693.2013.822604

Mettas, A., y Constantinou, C. P. (2008). The technology fair: A project-based learning approach for enhancing problem solving skills and interest in design and technology education. International Journal of Technology and Design Education, 18, 79-100. doi: 10.1007/s10798-006-9011-3

Mioduser, D., Nachmias, R. y Forkosh-Baruch, A. (2008). New Literacies for the knowledge society. En J. Voogt y G. Knezek (eds.). International Handbook of Information Technology in Primary and Secondary Education. Part One. (pp. 23-42). New York: Springer.

Mishra, P. y Koehler, M. (2006). Technological pedagogical content knowledge: A framework for teacher knowledge. Teachers College Record, 108,(6). 1017-1054. doi: 10.1111/j.1467-9620.2006.00684.x

Mishra, P., Koehler, M. J., y Henriksen, D. (2011). The 7 transdisciplinary habits of mind: Extending the TPACK framework towards 21st century learning. Educational Technology, 51(2), 22-28. Recuperado de: https://www.jstor.org/stable/44429913

Mishra, P. y Mehta, R. (2017). What we educators get wrong about 21st-century learning: Results of a survey. Journal of Digital Learning in Teacher Education, 33(1), 6-19. doi: 10.1080/21532974.2016.1242392

Murillo, F. J., y Krichesky, G. J. (2015). Mejora de la Escuela: Medio siglo de lecciones aprendidas. REICE. Revista Iberoamericana sobre Calidad, Eficacia y Cambio en Educación, 13(1), 69-102. Recuperado de https://bit.ly/2JlAh46

Osborne, J. y Hennessy, S. (2003). Literature Review in Science Education and the Role of ICT: Promise, Problems and Future Directions. 2003. Recuperado de: https://www.nfer.ac.uk/literature-review-in-science-education-and-the-role-of-ictpromise-problems-and-future-directions/. 
Overbay, A., Patterson, A., Vasu, E. y Grable, L. (2010). Constructivism and technology use: findings from the IMPACTing Leadership project. Educational Media International, 47, 2, 103-120. doi: 10.1080/09523987.2010.492675

Pozo, S., López, J., Moreno, A. J., y López, J. A. (2019). Impact of Educational Stage in the Application of Flipped Learning: A Contrasting Analysis with Traditional Teaching. Sustainability, 11(21), 1-15. doi:10.3390/su11215968

Pozo-Sánchez, S., López-Belmonte, J., Fernández, M. F., y López, J. A. (2020). Análisis correlacional de los factores incidentes en el nivel de competencia digital del profesorado. Revista Electrónica Interuniversitaria de Formación del Profesorado, 23(1), 143-151. https://doi.org/10.6018/reifop.396741

Prensky, M. (2001). Digital Natives, Digital Immigrants Part 1. On the Horizon, 9, 5. 1-6.

Sancho, J., Bosco, A., Alonso, C. y Sánchez, J. (2015). Formación del profesorado en Tecnología Educativa: de cómo las realidades generan los mitos. Revista Latinoamericana de Tecnología Educativa, 14(1), 17-30. doi: 10.17398/1695-288X.14.1.17

Sang, G., Valcke, M., Braak, J. V. y Tondeur, J. (2010). Student teachers' thinking processes and ICT integration: Predictors of prospective teaching behaviors with educational technology. Computers \& Education, 54(1), 103-112. doi: 10.1016/j.compedu.2009.07.010

Savage, J. (2010). A survey of ICT usage across English Secondary schools. Music Education Research, 12:1, 89-104. doi: 10.1080/14613800903568288

Snow, K. (2004). What counts as literacy in early childhood? En K. McCartney, y D. Phillips (Eds.), Blackwell Handbook of Early Childhood Development (p. 274-294). Malden, MA: Blackwell Publishing.

Tagle, T. (2011). El enfoque reflexivo en la formación docente. Calidad en la Educación, 34, 203215. http://dx.doi.org/10.4067/S0718-45652011000100011

Tejada, J. (2004). Música y mediación de la tecnología en sus procesos de aprendizaje. Educación XX1, 7.15-26. doi:10.5944/educxx1.0.7.327

Tejada, J. y Thayer, T. (2019a). Diseño, implementación y evaluación de una intervención de formación en tecnología musical basada en TPACK y ABP en la formación inicial del profesorado de música de Educación Secundaria. Revista Latinoamericana de Tecnología Educativa, 18(2). 9-30. doi:10.17398/1695-288X.18.2.9

Tejada, J. y Thayer, J. (2019b). Design and validation of a music technology course for initial music teacher education based on the TPACK Model and the Project-Based Learning approach. Journal of Music, Technology, and Education, 12, 3. 225-246 doi:10.1386/jmte_00008_1

Voogt, J., Knezek, G., y Somekh, B. (2008). 'Factors Affecting Teachers' Pedagogical Adoption of ICT. En J. Voogt and G. Knezek (eds.) International Handbook of Information Technology in Primary and Secondary Education (pp. 449-460). New York, NY: Springer. 